\title{
Comparison of Juvenile and Adult Onset Refractory Spondyloarthritis Patients Attending a Tertiary Rheumatology Clinic
}

\author{
MN ISLAM ${ }^{\mathrm{a} \& b}$, K ISLAM $^{\mathrm{c}}$
}

Summary:

Objectives: To compare the demographic and clinical characteristics of juvenile onset refractory spondyloarthritis (JOSpA) with a group of adult-onset refractory spondyloarthritis (AOSpA) patients.

Methods: In this real-life, cross-sectional study, a total of $215 S p A$ patients (refractory to two NSAIDs and/or DMARDs) were enrolled following ASAS classification criteria from a rheumatology center in Dhaka, Bangladesh. Based on age, the patients were divided into JOSpA group and AOSpA group for analysis. The patients' demographics, quality of life, laboratory and clinical characteristics were compared and analyzed between the two groups using chisquare and independent sample t-test.

Results: Among 215 patients, 57 (53 males, 4 females) were in JOSpA group, and 158 (126 males, 32 females) were in AOSpA group. Most patients were male in both groups $(\mathrm{P}=0.02)$. The mean age at diagnosis for JOSpA and AOSpA were $27.68 \pm 9.5$ and $40.28 \pm 10$ years respectively $(\mathrm{P}=0.00)$, and mean age at onset of disease in JOSpA and AOSpA were $12.33 \pm 3.62$ and $29.58 \pm 96.5$ years respectively. The mean disease duration was $15.36 \pm 9.3$ months in JOSpA compared

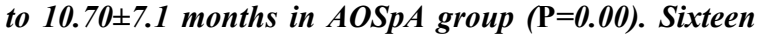
(28.1\%) JOSpA and 9 (5.7\%) AOSpA patients were undernourished, $(\mathrm{P}=0.00)$. On the contrary, obesity was more prevalent in AOSpA patients (41.1\%) than those in JOSpA patients (21.1\%) $(\mathrm{P}=0.00)$. There were significant differences

Introduction:

The spondyloarthritis ( $\mathrm{SpA}$ ) is a group of heterogeneous, immune-mediated, inflammatory arthritic diseases commonly affecting sacroiliac joints, spine,

a. Md. Nazrul Islam, Department of Rheumatology, Bangabandhu Sheikh Mujib Medical University (BSMMU).

b. Modern One Stop Arthritis Care and Research Center ${ }^{\circledR}$ (MOAC\&RC®), Dhaka, Bangladesh.

c. Khaleda Islam, Institute of Nutrition and Food Science, University of Dhaka.

Address of Correspondence: Prof. Md. Nazrul Islam, Professor of Rheumatology, Department of Rheumatology, Room No 1605, Level 16, D Block, Bangabandhu Sheikh Mujib Medical University, Dhaka. Mobile: 01678112396, Email: islam1nazrul@gmail.com

Received: 18 July 2019

Accepted: 22 January 2020 in mean haemoglobin $(11.38 \pm 1.7$ and $10.94 \pm 1.3 \mathrm{~g} / \mathrm{dl})$ $(\mathrm{P}=0.05)$ and mean serum creatinine $(0.94 \pm 0.2$ and $0.86 \pm 0.2$ mg/dl) $(\mathrm{P}=0.03)$ levels in AOSpA and JOSpA groups respectively. HLA-B27 was done in $20 \mathrm{JOSpA}$ and $61 \mathrm{AOSpA}$ patients. Among them, 19 (95\%) were positive in JOSpA and 55 (90.16\%) were positive in AOSpA groups. Similarly, hips involvement was higher in JOSpA group 19 (33.3\%) compared to the AOSpA group 21 (13.3\%) $(\mathrm{P}=0.004)$. Significant differences were observed in mean disease activity parameters, patient global assessment (PGA) (7.86 \pm 1.2 vs $7.38 \pm 1.4, \mathrm{P}=0.02), A S D A S-C R P(4.60 \pm 0.8$ vs $4.33 \pm 0.9$, $\mathrm{P}=0.05)$, and ASDAS-ESR (4.49 \pm 0.8 vs $4.17 \pm 1.0, \mathrm{P}=0.02)$ in JOSpA and AOSpA groups respectively. The modified Stoke AS Spinal Score (mSASSS) in AOSpA group was higher (24.18 \pm 17.22$)$ than that of JOSpA group (17.10 \pm 9.6$)$ $(\mathrm{P}=0.00)$.

Conclusions: In JOSpA patients, under nutrition, hip involvement, longer disease duration, uveitis, PGA, low Hb level and ASDAS-ESR disease activity scores were higher. On the contrary in AOSpA group, male gender, married subjects, obesity, higher creatinine level and mSASSS scores were higher.

Keywords: Juvenile-onset, Adult-onset, Refractory Spondyloarthritis

(J Bangladesh Coll Phys Surg 2020; 38: 79-85)

DOI: https://doi.org/10.3329/jbcps.v38i2.45631

entheses and peripheral joints. This frequently occurring chronic rheumatic diseases $(\mathrm{SpA})$ is known to have a strong association with the human leukocyte antigen (HLA-B27) ${ }^{[1,2]}$. According to The Assessment in SpondyloArthritis International Society (ASAS) criteria, SpA is classified as axial (nr-axial and axial) and peripheral $\mathrm{SpA}$. The disease can occur both in children and in adults. The onset of the disease during the childhood period ( $<16$ years) is known as Juvenile-onset Spondyloarthropathy (JOSpA) ${ }^{[3]}$. Some of them enter into adult $\mathrm{SpA}$ as a continuation of the childhood disease [4]. When the onset of SpA occurs $\geq 16$ years of age, it is known as adult-onset $\mathrm{SpA}{ }^{[5]}$.

The first-line treatment of SpA patients is non-steroidal anti-inflammatory drugs (NSAIDs). Most of the patients 
often become refractory to NSAIDs and/or DMARDs

${ }^{[6]}$. Once they become unresponsive to consecutive two NSAIDs, then they are classified as a case of refractory $\mathrm{SpA}^{[7]}$. Variability in the characteristics of disease as well as severity had been observed by authors both in JOSpA and AOSpA groups. According to some research studies, JOSpA patients may suffer from severe disease and co-morbidities such as psoriasis, inflammatory bowel disease (IBD), uveitis ${ }^{[8-10]}$, fever ${ }^{[11]}$, and autoimmune liver disease ${ }^{[12-13]}$. These differences might be more pronounced in refractory SpA patients. As such, they might play a significant role in the quality of life as well. Several studies were done in JOSpA and AOSpA patients to observe the differences in demographic and clinical manifestations as well as treatment outcome. Although there has been considerable progress in the field concerned with SpA ${ }^{[3,5]}$, there is still a dearth of sufficient information in regards to refractory spondyloarthritis ( $\mathrm{SpA})$. Refractory $\mathrm{SpA}$ features of juvenile-onset patients may continue to adulthood. Nevertheless, significant differences may exist in the presentation and clinical status of patients based on the age of onset. Till today, there has been no integral comparative investigation of refractory JOSpA and AOSpA patients.

This study was aimed to compare the differences in demographics, quality of life, laboratory and clinical characteristics between juvenile-onset and adult-onset refractory spondyloarthritis patients. The findings of the study are anticipated to enrich the existing literature on SpA.

\section{Methods:}

This was a real life, cross-sectional study, carried out among the refractory SpA patients attending for their medical care in a tertiary care rheumatology centre of Dhaka, Bangladesh.

\section{Subjects}

Informed written consent was taken from the participants before enrolment of this study. A total of 215 refractory SpA patients were enrolled from March 2015 to December 2017. The ASAS criteria were followed for the diagnosis of SpA. The diagnosed SpA patients were further screened to select refractory $\mathrm{SpA}$ group using a purposive sampling method. These selective patients who met the inclusion criteria were then divided into two groups based on the age of onset of $\mathrm{SpA}$ : juvenile-onset $\mathrm{SpA}$ (JOSpA) and adult-onset $\mathrm{SpA}$ (AOSpA) groups. For the convenience of analysis, patients, whose age of onset of $\mathrm{SpA}<18$ years were categorized as JOSpA group and e" 18 years as AOSpA group ${ }^{[11,14]}$.

\section{Questionnaire}

A semi-structured questionnaire was served during the face-to-face interview of the enrolled patients. The questionnaire was composed of 9 segments: (1) basic personal data; (2) biometrics information; (3) family history (relatives diagnosed with SpA); (4) AS and other SpA features such as, uveitis, psoriasis, inflammatory bowel disease (IBD); (5) laboratory results; (6) radiographic data- X-ray (7) disease activity score (Bath AS indices with a $0-10 \mathrm{~cm}$ visual analog scale (VAS), including the Bath AS Disease Activity Index (BASDAI), Bath AS Functional Index (BASFI), Bath AS Patient Global Assessment (BAS-G) and Bath AS Metrology Index (BASMI), Ankylosing Spondylitis Disease Activity Score with ESR (ASDAS-ESR) and Ankylosing Spondylitis Disease Activity Score with CRP (ASDAS-CRP); (8) Ankylosing Spondylitis Quality of Life (ASQoL) questionnaire and (9) vaccination history.

\section{Clinical evaluation}

A single trained medical officer recorded the demographics and clinical parameters in the semistructured questionnaire. It comprises of the duration of the disease, family history of low back pain, morning stiffness, extra-articular manifestations like uveitis, inflammatory bowel disease (IBD) and psoriasis as well as diagnosis of the subgroups of SpA.

A thorough physical examination of each patient was performed which includes pulse, blood pressure, BMI, 44 joints count, range of motion (ROM) of spine and assessment of enthesitis. The value estimated was then transferred to each item of the BASMI list of indices and PGA score was evaluated with a $0-10 \mathrm{~cm}$ VAS. The scores of BASDAI, pain visual analogue scale (VAS), $A S D A S-E S R$, and ASDAS-CRP were used to assess the disease activity. The BASFI and Bengali version of ASQoL were used to assess the functional status and quality of life respectively. The recorded information in the semi-structured questionnaire was randomly reviewed by a single treating rheumatologist. 


\section{Laboratory tests}

The performed laboratory studies were CBC, ESR, CRP, creatinine, SGPT and HLA-B27 using standard investigation methods. All the tests were done from the same pathology laboratory with the help of a single standard procedure. These measures were recorded on the day of the interview (present value).

\section{Radiographic tests}

$\mathrm{X}$-ray of the sacroiliac joint and lumbosacral spine and cervical spine (right lateral view) was done for assessment of modified Stoke AS Spinal Score (mSASSS). With the anteroposterior pelvis view, the sacroiliac joint was graded as 1-4; and anteroposterior and lateral views of the hip joints were used to assess hip arthritis. The radiological score was done by a single treating rheumatologist.

\section{Statistical analysis}

The different characteristics between JOSpA and AOSpA groups were compared with descriptive statistical analysis. The comparison of JOSpA and AOSpA groups were done with the help of categorical variables by using the Chi-square test or Fisher's exact test. The independent sample t-test was used to compare continuous variables. Statistical significance was considered when $\mathrm{p}$-value $<0.05$. All statistical analyses were carried out with SPSS software program version 20.0 (SPSS Inc., Chicago, IL, USA).

\section{Results:}

Among 215 patients, 57 (53 males, 4 females) were in JOSpA group, and 158 (126 males, 32 females) were in AOSpA group. There was a significant difference in the mean ages at the time of enrolment of JOSpA (27.68 \pm 9.5 years) and $\mathrm{AOSpA}(40.28 \pm 10$ years) groups respectively $(P=0.00)$.

No difference was observed in education, leaving area, family history, current diagnosis and quality of life in between two groups.

The demographic, disease duration, uveitis and quality of life of the two groups are shown in Table I.

Table-I

The demographic, disease duration, uveitis and Ankylosing Spondylitis Quality of Life of the study groups $(n=215)$

\begin{tabular}{llcccc}
\hline Characteristics & & JOSpA $(\mathrm{n}=57)$ & AOSpA $(\mathrm{n}=158)$ & $\mathrm{X}^{2}$ & P-value \\
\hline Age at onset of SpA & Mean (SD) & $12.33 \pm 3.62$ & $29.58 \pm 9.65$ & & \\
Gender & Male & $53(93.0 \%)$ & $126(79.7 \%)$ & $5.26^{*}$ & 0.02 \\
& Female & $4(7.0 \%)$ & $32(20.3 \%)$ & & \\
Marital Status & Married & $25(43.9 \%)$ & $140(88.6 \%)$ & $47.0^{*}$ & 0.00 \\
& Unmarried & $32(56.1 \%)$ & $18(11.4 \%)$ & & \\
BMI & Under-nutrition & $16(28.1 \%)$ & $9(5.7 \%)$ & $26.02^{*}$ & $0.00^{*}$ \\
& Normal & $23(40.4 \%)$ & $51(32.3 \%)$ & & \\
& Overweight & $6(10.5 \%)$ & $33(20.9 \%)$ & & \\
Disease duration (months) & $12(21.1 \%)$ & $65(41.1 \%)$ & & \\
H/O uveitis & Obese & $15.36 \pm 9.3$ & $10.70 \pm 7.1$ & & 0.00 \\
& Yes & $15(26.3)$ & $29(18.4)$ & $1.63^{*}$ & $0.20^{*}$ \\
ASQoL (0-18) & No & $42(73.7)$ & $129(81.6)$ & & \\
\hline
\end{tabular}

Independent sample t-test, * chi-square and Fisher's exact test, BMI- Body mass index 
Table II shows the laboratory and radiographic data of the two study groups. No difference was observed in WBC total count and differential count as well as platelet count in between groups.

Table-II

The laboratory and radiographic data of the study groups ( $n=215)$

\begin{tabular}{|c|c|c|c|c|c|c|}
\hline$\overline{\text { Data }}$ & Categories & $\operatorname{JOSpA}(\mathrm{n}=57)$ & $\operatorname{AOSpA}(n=158)$ & $\mathrm{X}^{2}$ & P-value & $\begin{array}{l}95 \% \text { Confidence } \\
\text { Interval }\end{array}$ \\
\hline $\mathrm{Hb}(\mathrm{gm} / \mathrm{dl})$ & & $10.94 \pm 1.3$ & $11.38 \pm 1.7$ & & 0.05 & $-0.87,-0.01$ \\
\hline ESR & & $68.98 \pm 25.1$ & $61.18 \pm 28.5$ & & 0.06 & $-0.18,15.77$ \\
\hline Serum creatinine $(\mathrm{mg} / \mathrm{dl})$ & & $0.86 \pm 0.2$ & $0.94 \pm 0.2$ & & 0.03 & $-0.15,-0.01$ \\
\hline SGPT & & $26.30 \pm 15.9$ & $30.55 \pm 20.2$ & & 0.15 & $-10.11,1.60$ \\
\hline CRP & & $72.49 \pm 59.7$ & $62.46 \pm 53.7$ & & 0.21 & $-6.82,26.88$ \\
\hline \multirow[t]{3}{*}{ HLA-B27 } & Positive & $19(33.3 \%)$ & $55(34.8 \%)$ & $0.44^{\#}$ & 0.80 & \\
\hline & Negative & $1(1.8 \%)$ & $6(3.8 \%)$ & & & \\
\hline & Not done & $37(64.9 \%)$ & $97(61.4 \%)$ & & & \\
\hline \multirow[t]{3}{*}{ Grade of Sacroiliitis } & Grade II & $0(0 \%)$ & $8(5.1 \%)$ & 5.24 & $0.06^{*}$ & \\
\hline & Grade III & $4(7 \%)$ & $23(14.6 \%)$ & & & \\
\hline & Grade IV & $53(93 \%)$ & $127(80.4 \%)$ & & & \\
\hline \multirow[t]{3}{*}{ Hip Involvement } & Yes & $19(33.3 \%)$ & $21(13.3 \%)$ & $11.31^{*}$ & 0.004 & \\
\hline & No & $15(26.3 \%)$ & $60(38.0 \%)$ & & & \\
\hline & Not evaluated & $23(40.4 \%)$ & $77(48.7 \%)$ & & & \\
\hline
\end{tabular}

Independent sample t-test, *chi-square and Fisher's exact test

The disease activity parameters in the two groups of patients are shown in Table III.

Table-III

The disease activity parameters of the study groups $(n=215)$

\begin{tabular}{lcccc}
\hline Characteristics & JOSpA $(\mathrm{n}=57)$ & AOSpA $(\mathrm{n}=158)$ & P-value & $\begin{array}{c}\text { 95\% Confidence } \\
\text { Interval }(\%)\end{array}$ \\
\hline Total tender joint & $3.89 \pm 4.8$ & $4.57 \pm 7.3$ & 0.43 & $-2.37,1.01$ \\
Total swollen joint & $1.07 \pm 2.0$ & $1.39 \pm 2.8$ & 0.44 & $-1.11,0.48$ \\
Back Pain & $7.02 \pm 2.17$ & $6.79 \pm 2.04$ & 0.48 & $-0.40,0.86$ \\
Morning stiffness & $3.40 \pm 3.70$ & $2.96 \pm 3.22$ & 0.43 & $-0.66,1.54$ \\
Peripheral pain/swelling & $5.37 \pm 3.200$ & $4.83 \pm 3.405$ & 0.30 & $-0.48,1.56$ \\
PGA & $7.86 \pm 1.2$ & $7.38 \pm 1.4$ & 0.02 & $0.06,0.90$ \\
BASMI & $4.89 \pm 2.5$ & $5.01 \pm 2.2$ & 0.74 & $-0.81,0.58$ \\
BASDAI & $5.97 \pm 1.9$ & $5.64 \pm 1.6$ & 0.20 & $-0.18,0.82$ \\
BASFI & $6.44 \pm 2.1$ & $6.04 \pm 2.0$ & 0.21 & $-0.22,1.01$ \\
mSASSS & $17.10 \pm 9.6$ & $24.18 \pm 17.22$ & 0.00 & $-11.45,-2.72$ \\
ASDAS-CRP & $4.60 \pm 0.8$ & $4.33 \pm 0.9$ & 0.05 & $-0.00,0.53$ \\
ASDAS-ESR & $4.49 \pm 0.8$ & $4.17 \pm 1.0$ & 0.02 & $0.06,0.59$ \\
\hline
\end{tabular}

Independent sample t-test, PGA-Patient global assessment, BASMI- Bath Ankylosing Spondylitis Metrology Index, BASDAI- Bath Ankylosing Spondylitis Disease Activity Index, BASFI- Bath Ankylosing Spondylitis Functional Index, ASDAS-Ankylosing Spondylitis Disease Activity Score, mSASSS- modified Stoke Ankylosing Spondylitis Spinal Score

\section{Discussion:}

This study explicates comprehensive information on the different clinical and demographic characteristics between the refractory JOSpA and AOSpA patients attending a tertiary care clinic in Bangladesh.
Various remarkable differences were illuminated by the study between the two groups of patients which include disease severity, clinical and demographic characteristics, indicators of inflammation, and radiographic findings of $\mathrm{SpA}$. Nonetheless, no 
significant distinction was observed in the family history of SpA, physical function, Bath AS scores and radiographic grading of sacroilitis among the two groups of patients. This corroborates with the study results observed by other researchers, Gensler et al. (2016) and O'Shea et al. (2009), who shared similar findings between patients of juvenile-onset ankylosing spondylitis (JOAS) and adult-onset ankylosing spondylitis (AOAS) ${ }^{[5,15]}$.

Both the study groups had a comparatively higher number of male patients (the male-to-female ratio being 3.9 in the AOSpA group and 13.2 in the JOSpA group) $(P<0.05)$. The male gender predominance in the study sample is consistent with other investigation findings, namely by Gensler et al. (2016) and O'Shea et al. (2009), who noted a higher number of male respondents in both juvenile-onset and adult-onset ankylosing spondylitis group of patients ${ }^{[5,15]}$. The prevalence of a positive family history of $\mathrm{SpA}$ in both the groups was high, indicating that a familial trait can present as a risk factor for the development of SpA in patients, who are more likely to seek medical attention either as an adult-onset or juvenile onset SpA. This corresponds with other research findings as well ${ }^{[5,15]}$. There were considerable distinctions in BMI between the two groups $(P<0.05)$. A comparatively high number of patients from JOSpA group presented with undernutrition, in contrast to patients from AOSpA group who showed a higher frequency of overnutrition. Undernourishment was also observed among JIA (Juvenile idiopathic arthritis) patients in another study by Ruperto et al. (2017) ${ }^{[14]}$, but this result was obtained from the median value of BMI which is inconsistent with the findings of this study, most likely due to different study settings and patient demographics.

The clinical profiles of the patients showed mark differences between the JOSpA and AOSpA groups in disease presentation. A high prevalence of axial SpA was recorded, compared to peripheral SpA in both the groups. Both axial and peripheral symptoms are more common among AOSpA patients than in JOSpA patients. A somewhat similar disease pattern was noted among the juvenile-onset (JOAS) and adult-onset Ankylosing Spondylitis (AOAS) patients in the research study by O'Shea et al. (2009), with the more common presentation of axial arthritis compared to peripheral arthritis in JOAS and AOAS ${ }^{[15]}$.
A distinguishing trend among JOSpA patients was to present with hip joint involvement, assumable due to longer disease duration. The mean age at onset of refractory SpA in JOSpA (12.33) and AOSpA (29.58) groups correspond to the mean age at onset of AS in adult and juvenile groups recorded in other studies ${ }^{[5,15-}$ 16]. Regarding extra-musculoskeletal manifestation, uveitis was more prevalent among JOSpA than AOSpA patients $(P>0.05)$. Comparatively, more JOAS patients were suffering from iritis in the study by Gensler et al. (2016) than AOAS patients ${ }^{[5]}$; this is also reflected in O'Shea et al.'s study where a higher percentage of JOAS patients were suffering from uveitis in comparison to AOAS patients ${ }^{[15]}$.

The highlight of this study entails a substantial comparison of various Bath AS indices, to determine the important differences in clinical and demographic characteristics between the JOSpA and AOSpA patients, who are refractory to a first-line $\mathrm{SpA}$ treatment. The high physical impairment was indicated among JOSpA patients by BASFI score in comparison to that in AOSpA patients, which had also been well observed in several studies ${ }^{[16-17]}$. This notion is further strengthened by a slightly higher ASQoL score and higher BASFI score among JOSpA patients compared to the AOSpA group, and this finding is further supported by the results of Ozgocmen et al.'s study ${ }^{[16]}$. Moreover, the JOSpA patients also showed elevated levels of CRP and ESR in their laboratory profile in contrast to their AOSpA counterpart, which had also shown in the systematic review by Jadon et al. [17]. Nonetheless, the corresponding ASDAS-CRP and ASDAS-ESR values also exhibited a notable difference between the two groups $(P<0.05)$. However, some studies revealed a lower BASFI, ASQoL and PGA scores among JOAS patients compared to the scores among AOAS patients $[15,18]$. This contrasting result can be attributed to the different patient characteristics in the population sample of the studies. In this study, the spondyloarthritic patients were refractory to NSAIDs and/or DMARDs indicating a late diagnosis of their current $\mathrm{SpA}$ conditions and consequently a poorer disease prognosis, particularly among JOSpA patients due to longer disease duration.

Both axial and peripheral SpA was found to be more prevalent in AOSpA group, but the corresponding hip involvement was relatively more common among JOSpA 
patients. Additionally, the disease severity assessed by PGA was reported to be higher among the JOSpA than the AOSpA group. A similar conclusion was derived by Jadon et al. ${ }^{17}$ and Duarte et al. ${ }^{18}$

JOSpA patients had a longer disease duration in comparison to AOSpA patients and had hip arthritis with a significantly more than that of axial arthritis (the mSASSS having a high significance level, $P<0.05$ ). This may present some inconveniences in the early diagnosis of JOSpA patients, as the axial involvement occurs at a later period of the disease duration ${ }^{19}$. Nonetheless, in this study, the JOSpA patients appeared slightly more impaired than those in AOSpA group which implies the necessity of early diagnosis and intervention for patients with JOSpA, particularly those who are already refractory to initial management.

The augmented disease severity with longer disease duration can be attributed to the possibility that these refractory $\mathrm{SpA}$ patients did not receive disease-state based specific therapeutic agents. The principal causes for this treatment delay may be due to limited availability of resources, physicians' apprehensiveness in using biologic agents, practitioners' attitude to disregard biologic agents' guidelines, and out-of-pocket medical expenditure of the patients.

Knowledge of the characteristic differences between the two refractory SpA patient groups in this study will be insightful and useful in determining a custom management guideline, catering to the individual characteristics of the refractory SpA patients. Erroneous patient information may prove to be a limitation in this study, due to the inaccurate recollection of symptoms and time period. In addition, the single source of study participants, i.e. tertiary care center, may give rise to a bias in the acquired data. Further investigation of the refractory SpA disease process detailing trigger factors, genetic predisposition and relationship between various variables are recommended. The future investigators can explore the likelihood of radiological damage impediment, with the help of early identification and treatment of refractory $\mathrm{SpA}$ cases. In a resourceconstrained country/setting, studies can search for appropriate disease-state initiation of treatment with late diagnosed cases of refractory SpA.

\section{Conclusions:}

The demographic, clinical, disease activity and laboratory characteristics are different between juvenile- onset and adult-onset refractory spondyloarthritis patients. In JOSpA patients, under nutrition, hip involvement, longer disease duration, uveitis, PGA, low $\mathrm{Hb}$ level and ASDAS-ESR disease activity scores were higher. A trend of slightly higher ASQoL, ESR, CRP and higher BASFI scores were in JOSpA patients as well. On the contrary, in AOSpA group, male gender, married subjects, obesity, higher creatinine level and mSASSS scores were higher. The JOSpA patients appeared slightly more impaired than those in AOSpA group which implies the necessity of early diagnosis and intervention particularly those who are already refractory to initial management. As a whole, these refractory SpA patients of both groups presented with augmented disease severity with longer disease duration. It could be for not been received disease-state based specific therapeutic agents. We assumed several reasons for this treatment delay like limited availability of resources, physicians' apprehensiveness in using biologic agents, practitioners' attitude to disregard biologic agents' guidelines, and out-of-pocket medical expenditure of the patients. Further large scale studies are needed to search factors playing role for such variation in presentation and outcome of JOSpA and AOSpA patients.

\section{Limitations}

Not all the patients were tested for HLA-B-27 (134 patients). A few patients were unable to provide the information on family history of $\mathrm{SpA}$ (42 patients). One hundred patients did not undergo investigation for hip involvement.

Acknowledgments: The authors would like to thank all participants of this study.

Funding: No funding was received from any source

Competing interests: None declared.

Author Contribution: Md. NI contributed in the conception and design of the study, data collections, analysis, data interpretation, drafting and KI contributed in analysis and editing of final manuscript.

\section{List of Acronyms:}

ASAS = Assessment of Spondyloarthritis International Society; ASDAS = Ankylosing Spondylitis Disease Activity Score; BASDAI = the Bath Ankylosing Spondylitis Disease Activity Index; BASFI = the Bath Ankylosing Spondylitis Functional Index; MRI = 
magnetic resonance imaging. NSAIDS $=$ Non-steroidal anti-inflammatory drugs; DMARDS= Disease-modifying antirheumatic drugs. $\mathrm{Nr}$-axial=non-radiographic axial.

ASQoL is a patient-reported outcome (PROs) tool consisting of a questionnaire which allows the rheumatologist to assess the functional outcome from the patient's perspective of the disease. The ASQoL questionnaire measures the quality of life with the help of scoring on a scale of $0-18$ (18 reflects the worst possible outcome in terms of functionality) ${ }^{[20]}$.

\section{References:}

1. Harja

ek M, Lamot L, Bukovac LT, Vidovi M, Joos R. Juvenile Spondyloarthritis: InTech; 2011.

2. Katsicas MM, Russo R. Biologic agents in juvenile spondyloarthropathies. Pediatric Rheumatology. 2016;14(1):17.

3. Burgos-Vargas R. Challenges in juvenile-onset spondyloarthritis. International Journal of Clinical Rheumatology. 2010;5(2):229.

4. Burgos-Vargas R. Juvenile onset spondyloarthropathies: therapeutic aspects. Archives of disease in childhood. 2003;88(4):312-8.

5. Gensler LS, Ward MM, Reveille JD, Learch TJ, Weisman $\mathrm{MH}$, Davis JC. Clinical, radiographic and functional differences between juvenile-onset and adult-onset ankylosing spondylitis: Results from the PSOAS cohort. Annals of the rheumatic diseases. 2008;67(2):233-7.

6. Rios Rodriguez V, Poddubnyy D. Tumor necrosis factor- \pm $(\mathrm{TNF} \pm)$ inhibitors in the treatment of nonradiographic axial spondyloarthritis: current evidence and place in therapy. Therapeutic advances in musculoskeletal disease. 2017;9(8):197-210.

7. Deodhar MRaA. Oxford Textbook of Axial Spondyloarthritis. Online: Oxford University Press; 2016 Oct 2016.

8. van der Horst-Bruinsma IE, Nurmohamed MT, Landewé RB. Comorbidities in patients with spondyloarthritis. Rheumatic Disease Clinics. 2012;38(3):523-38.

9. Dougados M, Wood E, Gossec L, Logeart I. Flare in axial spondyloarthritis: investigation of meaningful changes in symptomatic outcome measures. Clinical and experimental rheumatology. 2017;35(2):209-13.
10. Khan MA, Haroon M, Rosenbaum JT. Acute anterior uveitis and spondyloarthritis: more than meets the eye. Current rheumatology reports. 2015;17(9):59.

11. Cherqaoui B, Rossi-Semerano L, Georgin-Lavialle S, Dusser P, Galeotti C, Piram M, et al. Boundaries between familial Mediterranean fever and juvenile spondyloarthritis: Analysis of three French retrospective cohorts. Joint Bone Spine. 2018.

12. Selmi C, Generali E, Gershwin ME. Rheumatic manifestations in autoimmune liver disease. Rheumatic Disease Clinics. 2018;44(1):65-87.

13. de Moll EH, Chang MW, Strober B. Psoriasis in adults and children: Kids are not just little people. Clinics in dermatology. 2016;34(6):717-23.

14. Ruperto N, Brunner HI, Zuber Z, Tzaribachev N, Kingsbury DJ, Foeldvari I, et al. Pharmacokinetic and safety profile of tofacitinib in children with polyarticular course juvenile idiopathic arthritis: results of a phase 1, open-label, multicenter study. Pediatric Rheumatology. 2017;15(1):86.

15. O'Shea FD, Boyle E, Riarh R, Tse SM, Laxer RM, Inman RD. Comparison of clinical and radiographic severity of juvenile-onset versus adult-onset ankylosing spondylitis. Annals of the rheumatic diseases. 2009;68(9):1407-12.

16. Ozgocmen S, Ardicoglu O, Kamanli A, Kaya A, Durmus B, Yildirim K, et al. Pattern of disease onset, diagnostic delay, and clinical features in juvenile onset and adult onset ankylosing spondylitis. The Journal of rheumatology. 2009;36(12):2830-3.

17. Jadon DR, Ramanan AV, Sengupta R. Juvenile versus adultonset ankylosing spondylitis-clinical, radiographic, and social outcomes. a systematic review. The Journal of rheumatology. 2013;40(11):1797-805.

18. Duarte AP, Marques CD, Bortoluzzo AB, Gonçalves CR, Silva JABd, Ximenes AC, et al. Epidemiologic profile of juvenile-onset compared to adult-onset spondyloarthritis in a large Brazilian cohort. Revista brasileira de reumatologia. 2014;54(6):424-30.

19. Shirley M, Laxer RM. New advances in juvenile spondyloarthritis. Nature Reviews Rheumatology. 2012;8(5):269.

20. López-Medina C, Garrido-Castro JL, Castro-Jiménez J, González-Navas C, Calvo-Gutiérrez J, Castro-Villegas MC, et al. Evaluation of quality of life in patients with axial spondyloarthritis and its association with disease activity, functionality, mobility, and structural damage. Clinical rheumatology. 2018; 37:1581-8. 